\title{
Association of Age and Serum Vitamin D Levels in Men with Metabolic Syndrome
}

\author{
Dina M. Qahwaji \\ Department of Clinical Nutrition, Faculty of Applied Medical Sciences, King Abdulaziz University, Jeddah, Saudi Arabia
}

\begin{abstract}
Objective: To determine the association of age and serum vitamin D levels in men with (Mets) and without (NMetS) metabolic syndromes.

Study Design: Descriptive study.

Place and Duration of Study: King Abdulaziz University Hospital (KAUH), Jeddah, Kingdom of Saudi Arabia (KSA), from January 2019 to December 2020.

Methodology: Properly diagnosed male subjects with MetS [n: 113 (49.13\%); age: 26-60 years], and age-matched control subjects with NMetS [n:117 (50.87\%); age: 26-60 years] were studied for the determination of serum vitamin D (vitD) levels, and correlation of age and serum vitD.

Results: Non-significant change in serum vitD levels were obtained in MetS compared to NMetS ( $p>0.05$ ) for whole data; as well as in MetS compared to NMetS subjects for all age-range groups. Insufficiency of serum vitD was found in both MetS and NMets subjects. However, mean values for MetS and NMetS showed age-wise lowering in vitamin $D$ and highly significant negative linear correlation of age and vitD for MetS (R: $-0.508, p<0.001)$ and NMetS (R: $-0.522, p<0.001$ ).

Conclusion: Present report, emphasising the significant negative linear correlation of age with serum vitD levels in MetS and NMetS subjects, provides potential information for understanding the discomforts caused by the insufficiency of vitamin D with the increase in age.
\end{abstract}

Key Words: Serum vitD, Age, Metabolic syndrome, Age-vitD association.

How to cite this article: Qahwaji DM. Association of Age and Serum Vitamin D Levels in Men with Metabolic Syndrome. J Coll Physicians Surg Pak 2022; 32(03):298-302.

\section{INTRODUCTION}

Metabolic syndrome (MetS) mainly causes diabetes mellitus (DM) and cardiovascular disease (CVD), ${ }^{1,2}$ as an influence of obesity, hypertension, hyperglycemia, dyslipidemia and a variety of other factors. ${ }^{2}$ Being overweight and obese is also linked with the inappropriate diet intake ${ }^{3}$ that may lead to MetS. Metabolic syndrome is an aggregation of various components showing an increase in the risk of CVD and type-2 diabetes mellitus (T2DM) and all-cause mortality directly due to interconnected clinical, physiological, biochemical and metabolic factors. ${ }^{4}$

There are a number of factors that might be associated with MetS, including visceral adiposity, insulin resistance, hyperglycemia, genetic factors, high blood pressure (BP), chronic stress, hypercoagulable state, dyslipidemia (mainly reduced high density lipoprotein cholesterol (HDL-C), elevated triglycerides (TG) and endothelial dysfunction. ${ }^{4}$

Correspondence to: Dr. Dina M. Qahwaji, Department of Clinical Nutrition, Faculty of Applied Medical Sciences, King Abdulaziz University, Jeddah, Saudi Arabia E-mail: dqahweji@kau.edu.sa

Received: August 18, 2021; Revised: November 20, 2021; Accepted: January 05, 2022

DOI: https://doi.org/10.29271/jcpsp.2022.03.298
Vitamin D (vitD), in this context, is an important factor that might be involved in MetS. Age-based variations in vitD levels in subjects with 20-74 years-age showed the prevalence of MetS, central obesity, and impaired fasting blood glucose level as $23.5 \%, 50,6 \%$ and $20.3 \%$, respectively. ${ }^{5}$ However, vitD variations at various age range values were not documented. ${ }^{5} \mathrm{Simi}-$ larly, age range-based studies for vitD variations in MetS are scarce in children, adults or elderly subjects, though the men with MetS had variations in serum vitD more pronounced than women of age range $16-79$ years ${ }^{6}$ and 65 years age or older. ${ }^{7}$

Type-2 diabetic model rat studies showed significant amelioration of glucose and lipid profile by vitamin $\mathrm{D}_{3}$ analogs-mediated modulation, ${ }^{8}$ and it was found that vitD representing bone and calcium status correlates inversely with body fat mass, lipid metabolism, BP and glucose regulation. Impaired metabolism may cause muscle damage; whereas, vitD influences trophic muscle functions. ${ }^{9}$ Overweight and obesity status is associated with vitD. ${ }^{10,11}$ These and other studiespresent the relationship of serum vitD (D-25(OH)D) and MetS. ${ }^{12,13}$

MetS is quite prevalent in Saudi Arabia, mainly involving cardiac diseases and diabetes mellitus. Vitamin $D$ deficiency may influence various features in MetS, though the precise role of vitD in various age groups in subjects with metabolic syndrome is still not known. Hence, this study was planned to determine 
association of advancing age on serum vitD levels in male subjects with MetS by comparing with the age-matched nonmetabolic syndrome (NMetS) control subjects.

\section{METHODOLOGY}

This descriptive study was conducted at King Abdulaziz University Hospital (KAUH), Jeddah, Kingdom of Saudi Arabia (KSA), from January 2019 to December 2020. Clinical examination and general tests/evaluations were carried out in the present work for dividing the subjects into MetS and NMetS groups. The MetS was defined with the special mention of the risk of CVD and type-2 diabetes mellitus (T2DM) as recommended previously. ${ }^{1,2,4}$ The NMetS group of subjects served as a control group. Any subject with current treatment/supplementing with vitD or calcium or drugs for the management of osteoporosis/osteopenia or a long history of having osteoporosis was excluded.

The MetS and NMetS subjects were diagnosed on the basis of measuring body mass index (BMI; weight in kilograms (Kgs) divided by respectivemeterssquared $\left(\mathrm{m}^{2}\right)$ height), ${ }^{14}$ waist-circumference (WC), ${ }^{15}$ blood pressure (BP)-systolic (SBP) and diastolic (DBP) using mercury sphygmomanometer, ${ }^{16}$ fasting blood glucose (FBG), ${ }^{17}$ serum HDL-C ${ }^{18}$ and serum triglycerides (TG). ${ }^{19}$ Waist circumference (WC) was assessed by measuring at iliac crest, while the patients in standing position. The BP measurements were carried out twice from the right arm after a rest period of 15 minutes in sitting position. Diabetes mellitus (DM) was defined as having FBG $>126 \mathrm{mg} / \mathrm{dl}$ or self-reporting as having DM, and vitD deficiency was defined by the serum vitD or $25-(\mathrm{OH}) \mathrm{D}$ $<20 \mathrm{ng} / \mathrm{ml}$, and vitD insufficiency defined by $20-29 \mathrm{ng} / \mathrm{ml}$ serum levels. $^{20}$

Diagnostic characterisation of MetS subjects and NMetS control subjects based on standard criteria, ${ }^{21}$ considering the presence of three or more of the features $\mathrm{HDL}-\mathrm{C}<50 \mathrm{mg} / \mathrm{dl}$, WC $\geq 88 \mathrm{~cm}$, FBG $>110 \mathrm{mg} / \mathrm{dl} /$ or antidiabetic treatment, SBP and DBP as $\geq 130$ and $\geq 85 \mathrm{mmHg} /$ or antihypertensive treatment, triglycerides (fasting) $\geq 150 \mathrm{mg} / \mathrm{d}$, respectively. The subjects showing the presence of two or less of the mentioned tests were considered as control or NMetS subjects.

All subjects in both groups (MetS, n: 113, NMetS, 117) with age range (26-60 years) were subdivided into age-based six sub-groups (26-30, 31-35, 36-40, 41-45, 46-50, 51-55, 56-60). Determination of serum vitD was carried out in age-matched male subjects with and without MetS. The ELISA technique was used for the determination of serum vitD.

The data for the present study was collected from the Outpatients Department at the Hospital. Data sheets were filled up with the supervision of a cardiologist. The consent of patients was obtained before the start of the study. All questions relating to descriptive measures were recorded. Blood samples were analysed. Ethical approval letter was obtained from the Ethical Approval Committee (Ref. No. 47-19).

The frequency and percentage data of descriptive statistics were calculated for qualitative variables, and mean \pm SD were used for quantitative variables. The unpaired ' $t$ ' test was applied for analysing two-tailed $p$ values for age and serum vitD levels to determine the statistical difference. Comparison of \% subjects of MetS and NMetS in various age range-groups was done using Chisquare test, analysed by MedCalc software. Significance evaluation relied on $p<0.05$.

The SPSS version 28 software was used for data entry and statistical analysis. Regression was used to find the cause-and-effect relationship between age of the subjects and serum vitD by employing an equation. The scatterplot of the points showed the strength of correlation. Analysis of the correlation coefficient $\mathrm{R}$ was used to measure the correlation between age and serum vitD in MetS and NMetS subjects.

\section{RESULTS}

The vitD serum levels in men having MetS (age: $44.39 \pm 9.85$ years) were compared with those in age-matched NMetS (age: $44.43 \pm 9.94$ years). Total subjects in the present study were 230, though additional 32 subjects had too high or too low levels of serum vitD, and hence, were excluded. The MetS subjects were $113(49.13 \%)$ and NMetS subjects were 117 (50.87\%). The mean \pm SD values for serum vitD in MetS subjects (24.75 \pm 12.26$)$ did not differ statistically from the NMetS subjects $(24.58 \pm 12.02, p$ : 0.916 , Table I), though these levels showed insufficiency of serum vitD.

The mean \pm SD values for age (years) range and serum vitD levels for the subjects with MetS and NMetS are given in Table I. The comparison of mean \pm SD of the vitamin D levels showed no significant decrease in vitD in MetS as compared to NMetS for all agerange groups ( $p>0.05$, Table I). However, mean values were found gradually decreasing (though non-significantly) from lower age range subjects to higher age range subjects in MetS as well as in NMetS. The vitD levels in \% subjects of MetS and NMetS in various age range-groups did not differ statistically (Chi-square test, $p>0.05$ ).

The subjects with MetS showed a highly significant negative linear correlation between age and vitD (R: -0.508, $p<0.001$, Table II). The subjects with NMetS also showed a highly significant negative linear correlation between age and vitD (R: $-0.522, p<0.001$, Table II).

Association of age and vitamin D in MetS and NMetS subjects for age-range groups showed negative linear; but non-significant correlation except for the age range group 31-35 that gave the $R$ valueas -0.7065 for NMetS ( $p<0.007$, Table II). Age group 31-35 in MetS showed non-significant negative linear correlation, though close to significance (R: $-0.516, p<0.071$, Table II) as compared to all other age range groups.

\section{DISCUSSION}

Insufficiency of serum vitD and highly significant negative linear correlation of age and vitD for subjects with and without metabolic syndrome is quite similar to other reports, ${ }^{8,10,11,20,22}$ though the inverse relationship of serum vitD and MetS has also been presented. ${ }^{12}$ The potential aspect in the present study, however, relates to investigating the unknown variations in vitD levels in various age range groups of subjects with metabolic syndrome. 
Table I: Serum vitamin D levels in subjects with MetS and NMetS.

\begin{tabular}{|c|c|c|c|c|c|c|c|}
\hline \multirow{4}{*}{ Age (years) range groups of subjects } & \multicolumn{7}{|c|}{ Serum VitD levels in subjects with MetS and NMetS (mean \pm SD) } \\
\hline & \multicolumn{3}{|c|}{\begin{tabular}{|c|} 
MetS \\
\end{tabular}} & \multicolumn{3}{|c|}{ NMetS } & \multirow{3}{*}{ p-value } \\
\hline & \multicolumn{2}{|c|}{ Subjects } & \multirow{2}{*}{$\begin{array}{c}\text { VitD levels } \\
\text { (ng/ml) }\end{array}$} & \multicolumn{2}{|c|}{ Subjects } & \multirow{2}{*}{$\begin{array}{c}\text { VitD levels } \\
(\mathrm{ng} / \mathrm{ml})\end{array}$} & \\
\hline & $\mathbf{n}$ & $\%$ & & $\mathbf{n}$ & $\%$ & & \\
\hline $26-30$ & 13 & 11.50 & $34.65 \pm 13.01$ & 14 & 11.97 & $33.77 \pm 13.45$ & 0.864 \\
\hline $31-35$ & 13 & 11.50 & $33.30 \pm 11.21$ & 13 & 11.11 & $33.40 \pm 9.76$ & 0.981 \\
\hline $36-40$ & 14 & 12.39 & $28.14 \pm 9.90$ & 14 & 11.97 & $28.39 \pm 9.70$ & 0.947 \\
\hline $41-45$ & 16 & 14.16 & $24.78 \pm 11.36$ & 17 & 14.53 & $24.49 \pm 11.55$ & 0.943 \\
\hline $46-50$ & 19 & 16.81 & $22.11 \pm 11.50$ & 19 & 16.24 & $23.01 \pm 11.77$ & 0.814 \\
\hline $51-55$ & 19 & 16.81 & $19.99 \pm 12.06$ & 20 & 17.09 & $19.17 \pm 11.14$ & 0.827 \\
\hline $56-60$ & 19 & 16.81 & $17.01 \pm 7.42$ & 20 & 17.09 & $16.71 \pm 6.45$ & 0.893 \\
\hline $26-60$ & 113 & 100 & $24.75 \pm 12.26$ & 117 & 100 & $24.58 \pm 12.02$ & 0.916 \\
\hline
\end{tabular}

Table II: Correlation of age range and vitamin D in MetS and NMetS subjects.

\begin{tabular}{|c|c|c|c|c|}
\hline \multirow{3}{*}{$\begin{array}{l}\text { Age (years) range of } \\
\text { subjects }\end{array}$} & \multicolumn{4}{|c|}{ Correlation coefficient $(R)$ for age and serum vitD in subjects with MetS and NMetS } \\
\hline & \multicolumn{2}{|c|}{ MetS } & \multicolumn{2}{|c|}{ NMetS } \\
\hline & $\mathbf{R}$ & p-value & $\mathbf{R}$ & p-value \\
\hline $26-30$ & -0.003 & 0.993 & -0.151 & 0.606 \\
\hline $31-35$ & -0.516 & 0.071 & -0.707 & $0.007^{* *}$ \\
\hline $36-40$ & -0.464 & 0.094 & -0.276 & 0.340 \\
\hline $41-45$ & -0.076 & 0.778 & -0.079 & 0.763 \\
\hline $46-50$ & -0.028 & 0.908 & -0.129 & 0.599 \\
\hline 51-55 & -0.389 & 0.100 & -0.328 & 0.158 \\
\hline $56-60$ & -0.023 & 0.927 & -0.083 & 0.729 \\
\hline $26-60$ & -0.508 & $0.001 * *$ & -0.522 & $0.001^{* *}$ \\
\hline
\end{tabular}

Age-range based comparative studies for serum vitD carried out in the current study are scarce in literature. The investigators who carried out studies in the age range of 20-74 years, 16-79 years and 65 years and above did not mention

In the present study, serum vitD levels were insufficient in both MetS and NMetS, which is in accordance with the definition of the insufficiency of serum vitD levels at 20-29 $\mathrm{ng} / \mathrm{ml} .^{20}$ It can appropriately be explained with the help of other reports ${ }^{8,10,11,22}$ and may further be interpreted with the investigation that vitD influences the trophic functions. ${ }^{22}$ Furthermore, the overweight and obesity status associated with vitD deficiency via the expression of genes that encode enzymes for controlling vitD in adipocytes, ${ }^{11}$ i.e. people increasing their body fats get decrease in vitD metabolism in view of vitD decomposition as well as decrease in the involved activating enzymes resulting to negative effect on the synthesis of vitD.

The present work was carried out exclusively in men aged 26-60 years. However, this comparison emphasises the need of studying the involvement of vitD in MetS and NMetS subjects of various age range groups in adult men, women, children and the elderly. In this respect, the opportunity of studying subjects with various age groups in the current report seems quite helpful for future potential studies. the comparison of vitD at various age range groups..$^{5-7}$ Hence, the present work provides further information for the role of vitD in subjects with MetS and NMetS control subjects.

Obesity status described in another report is similar to the present work in certain perspectives since inverse correlation of vitD and insulin resistance was investigated as a major factor in obese and over-weight adult subjects. ${ }^{13}$

Results of the involvement of vitD levels in subjects (with various age-range groups) with MetS and without MetS obtained in the present work can be interpreted by several investigations. ${ }^{22-25}$ It was explained with animal models, association with MetS, influence on serum vitD and effect of supplementation of vitD to model MetS rats, ${ }^{23,24}$ damage / inflammation leading to MetS ${ }^{22}$ and consideration of vitD deficiency as one of the important risk factors in MetS. ${ }^{22,25}$

Considering the present study, it is suggested that obesity status and other features in subjects with/without metabolic syndrome may influence other factors considered as the risk factors of MetS. These features may further lead to decrease the serum HDL-C- the main diagnostic feature and quite common risk factor of MetS. Furthermore, obesity showing quite high waist circumference (WC) and BMI and decrease 
in HDL-C may accompany the other major risk factors of MetS including high BP, hyperglycemia and high serum triglycerides that may keep converting the NMetS status to MetS status. Hence, proper management for the MetS risk factors is helpful for the patients with/without MetS.

The present study is an attempt to investigate the association between age and vitD levels in MetS and NMetS subjects. However, the age range of the whole data (MetS/NMetS subjects) requires to be widened, especially onward from 60 years to 70 or 75 years, in male and female subjects. This might clarify the complex role of age-based serum vitamin $D$ variations.

\section{CONCLUSION}

The present report emphasising the significant negative linear association of age with the serum vitD levels in MetS and NMetS subjects provides potential information for understanding the discomforts caused by the deficiency or insufficiency of vitD with increase in age.

Metabolic syndrome in Saudi Arabia is quite prevalent, and hence, it is urgently required to have and create further awareness in the general public for proper management of MetS.

\section{ETHICAL APPROVAL:}

Ethical approval was granted by the Unit of Biomedical Ethics Research Committee in Medical Department, King Abdulaziz University, KSA, on January 22, 2019.

\section{PATIENTS' CONSENT:}

All subjects/patients voluntarily signed informed consent.

\section{CONFLICT OF INTEREST:}

The author declared no conflict of interest.

\section{AUTHOR'S CONTRIBUTION:}

The author solely contributed all aspects of the manuscript.

\section{REFERENCES}

1. Cheng L, Yan W, Zhu L, Chen Y, Liu J, Xu Y, et al. Comparative analysis of IDF, ATPIII and CDS in the diagnosis of metabolic syndrome among adult inhabitants in Jiangxi Province. China. PLoS One 2017; 12(12):e0189046. doi: 10.1371/journal.pone.0189046.

2. Mbugua SM, Kimani ST, Munyoki G. Metabolic syndrome and its components among university students in Kenya. $B M C$ Public Health 2017; 17(1):909. doi: 10.1186/s12889. 017-4936-x.

3. Almeda-Valdes P, Herrera-Mercadillo RJ, Aguilar-Salinas CA, Uribe $M$, Méndez-Sánchez $N$. The role of diet in patients with metabolic syndrome. Curr Med Chem 2019; 26(19):3613-9. doi: 10.2174/0929867324666170518095316.
4. Kaur J. A comprehensive review on metabolic syndrome. Cardiol Res Pract 2014; 2014:943162. doi: 10.1155/2014/ 943162.

5. Ghadieh R, Mattar Bou Mosleh J, Al Hayek S, Merhi S, El Hayek Fares J. The relationship between hypovitaminosis D and metabolic syndrome: A cross sectional study among employees of a private university in Lebanon. BMC Nutr 2018; 4:36. doi: 10.1186/s40795-018-0243-x.

6. García-Bailo B, Da Costa LA, Arora P, Karmali M, El-Sohemy A, Badawi A. Plasma vitamin D and biomarkers of cardiometabolic disease risk in adult Canadians, 2007-2009. Prev Chronic Dis 2013; 10:E91. doi: 10.5888/pcd10.120230.

7. Shoben AB, Kestenbaum B, Levin G, Hoofnagle AN, Psaty $B M$, Siscovick DS, et al. Seasonal variation in 25 hydroxyvitamin $D$ concentrations in the cardiovascular health study. Am J Epidemiol 2011; 174(12):1363-72. doi: 10.1093/aje/kwr258.

8. Abdel-Rehim WM, El-Tahan RA, El-Tarawy MA, Shehata RR, Kamel MA. The possible antidiabetic effects of vitamin D receptors agonist in rat model of type 2 diabetes. Mol Cell Biochem 2019; 450(1-2):105-112. doi: 10.1007/s11010018-3377-x.

9. Trovato FM, Castrogiovanni P, Szychlinska MA, Purrello F, Musumeci G. Early effects of high-fat diet, extra-virgin olive oil and vitamin D in a sedentary rat model of non-alcoholic fatty liver disease. Histol Histopathol 2018; 33(11): 1201-13. doi: 10.14670/HH-18-008.

10. Cimini FA, Barchetta I, Carotti S, Bertoccini L, Baroni MG, Vespasiani-Gentilucci $U$, et al. Relationship between adipose tissue dysfunction, vitamin D deficiency and the pathogenesis of non-alcoholic fatty liver disease. World J Gastroenterol 2017; 23(19):3407-17. doi: 10.3748/wjg. v23.i19.3407.

11. Seldeen $K L$, Pang $M$, Rodríguez-Gonzalez $M$, Hernandez $M$, Sheridan $Z, Y u P$, et al. A mouse model of vitamin D insufficiency: Is there a relationship between $25(\mathrm{OH})$ vitamin D levels and obesity? Nutr Metab (Lond) 2017; 14:26. doi: 10.1186/s12986-017-0174-6.

12. Snijder MB, Lips P, Seidell JC, Visser M, Deeg DJ, Dekker JM, et al. Vitamin D status and parathyroid hormone levels in relation to blood pressure: A population-based study in older men and women. J Intern Med 2007; 261(6): 558-65. doi: 10.1111/j.1365-2796.2007.01778.x.

13. Choi HS, Kim KA, Lim CY, Rhee SY, Hwang YC, Kim KM, et al. Low serum vitamin $D$ is associated with high risk of diabetes in Korean adults. J Nutr 2011; 141(8):1524-8. doi: 10.3945/jn.111.139121.

14. Keys A, Fidanza F, Karvonen MJ, Kimura N, Taylor $\mathrm{HL}$. Indices of relative weight and obesity. J Chronic Dis 1972; 25(6):329-43. doi: 10.1093/ije/dyu058.

15. Litchford MD. Clinical: Biochemical, physical, and functional assessment. In: Mahan LK, Raymond J, Eds. Krause's Food \& The Nutrition Care Process. ed. 14th.Missouri: Elsevier; 2017: p. 114.

16. Oh SY, Ryue J, Hsieh CH, Bell DE. Eggs enriched in omega-3 fatty acids and alterations in lipid concentrations in plasma and lipoproteins and in blood pressure. Am J Clin Nutr 1991; 54(4):689-695. doi: 10.1093/ajcn/54.4.689.

17. Dacus J, Schulz K, Averill A, Sibai B. Comparison of capillary 
Age-based vitamin D levels in men with metabolic syndrome

accu-chek blood glucose values to laboratory values. Am J Perinatol 1989; 6(3):334-6. dol: 10.1055/s-2007-999608

18. Warnick GR, Nguyen T, Alders A. Comparison of improved precipitation methods for quantification of high-density lipoprotein cholesterol. Chin Chem 1985; 31(2):217-22.

19. Nagele U, Haggle EO, Saver G, Wiedemann E, Lehmann P, Wahlefeld AW, et al. Reagent for the enzymatic determination of serum total triglycerides with improved lipolytic efficiency. J Chin Chem Chin Biochem 1984; 22(2): 165-74. dol: 10.1515/cclm.1984.22.2.165.

20. Institute of Medicine (IOM). Dietary reference intakes for calcium and vitamin D. Washington DC: The National Academies Press; 2011.

21. The expert panel on detection, evaluation, and treatment of high blood cholesterol in adults. Executive summary of the third report of the national cholesterol education program (NCEP) expert panel on detection, evaluation, and treatment of high blood cholesterol in adults (Adult treatment panel III). JAMA 2001; 285(19):2486-97. dol: 10.1001/jama.285.19.2486.

22. Trovato FM, Castrogiovanni P, Szychlinska MA, Purrello F,
Musumeci G. Impact of western and mediterranean diets and vitamin $d$ on muscle fibers of sedentary rats. Nutrients 2018; 10(2):231. dol: 10.3390/nu10020231.

23. Mostafa DK, Nasa RA, Zahran N, Ghoneim MT. Erratum to 'Pleiotropic protective effects of vitamin $D$ against high fat diet-induced metabolic syndrome in rats: One for all' [Eur. J. Pharmacol 2016; 792: 38-47]. Eur J Pharmacol 2017; 806:110. dol: 10.1016/j.ejphar.2017.03.020.

24. Drori A, Rotnemer-Golinkin D, Avi S, Drori A, Danny O, Levanon $\mathrm{D}$, et al. Attenuating the rate of total body fat accumulation and alleviating liver damage by oral administration of vitamin D-enriched edible mushrooms in a diet-induced obesity murine model is mediated by an antiinflammatory paradigm shift. BMC Gastroenterol 2017; 17(1):130. dol: 10.1186/s12876-017-0688-4.

25. Kim HS, Kim TH, Lee HH, Kim SK, Lee B, Park YH. Relationship between Serum 25-hydroxy-vitamin D concentration and risk of metabolic syndrome in patients with fatty liver. J Bone Metal 2017; 24(4):223-8. do: 10.11005/jbm.2017.24.4.223.

302

Journal of the College of Physicians and Surgeons Pakistan 2022, Vol. 32(03): 298-302 